Article

\title{
DegoViz: An Interactive Visualization Tool for a Differentially Expressed Genes Heatmap and Gene Ontology Graph
}

\author{
Somyung $\mathrm{Oh}^{1}$, Junghyeon $\mathrm{Ha}^{2}$, Kyungwon Lee ${ }^{2}$ and Sejong $\mathrm{Oh}^{3, *}$ \\ 1 Department of Visualization, Texas A\&M University, College Station, TX 77843, USA; \\ somyungoh@gmail.com \\ 2 Department of Digital Media, Ajou University, Suwon 16499, Korea; hajh0104@gmail.com (J.H.); \\ swissrain@gmail.com (K.L.) \\ 3 Department of Software Science, Dankook University, Yongin 16890, Korea \\ * Correspondence: sejongoh@dankook.ac.kr; Tel.: +82-31-8005-3222
}

Academic Editor: Hung Nguyen

Received: 27 March 2017; Accepted: 25 May 2017; Published: 25 May 2017

\begin{abstract}
Microarray is a general scheme to identify differentially expressed genes for a target concept and can be used for biology. The output is presented utilizing a heatmap that biologists analyze in related terms of gene ontology to determine the characteristics of differentially expressed genes. In this paper, we propose an integrated visualization tool for a heatmap and gene ontology graph. Most of the previous methods used were static and none of them were combined. The proposed visualization tool integrates these and provides users with an interactive management ability. Users can easily identify and confirm related terms of gene ontology for given differentially expressed genes. Further, the proposed tool visualizes the connections between genes on the heatmap and gene ontology graph. Therefore, the proposed tool can be used for precision healthcare.
\end{abstract}

Keywords: differentially expressed gene; gene ontology; heatmap; microarray; visualization

\section{Introduction}

Genes are related to many life phenomena. Researchers attempt to determine casual genes related to disease or other life phenomena. If we are able to identify useful information from genes, we can apply it to genomic medicine or healthcare training; precision healthcare may be possible. To accomplish this, we compare the gene expression level between case and control samples. If differentially expressed genes (DEGs) exist between the case and control samples, they are considered candidates of causal genes. Microarray is a general scheme employed to identify differentially expressed genes for a target concept [1]. In the microarray image, each dot corresponds to an oligonucleotide probe and its color indicates the expression level of the gene. Image analysis software converts the colors to numeric expression values. RNA-Seq is another scheme used to locate DEGs. It was developed for transcriptome profiling. It provides a considerably more precise measurement of the levels of transcripts and their isoforms than other next generation sequencing (NGS) methods [2].

A DEG list consists of a gene list and their expression values. However, it is difficult to understand the meanings of a gene set. Therefore, a heatmap is used to visualize DEGs. In a heatmap, a column indicates the expression levels of a gene and a row indicates a sample.

Even though the heatmap indicates the difference in the gene expression levels between two groups, biologists must determine the characteristics of the genes in a DEG list. We pose the following questions. What are the common characteristics of upregulated genes? What is the difference between 
upregulated and downregulated genes? Gene ontology (GO) [3] is widely used to determine the characteristics of a given gene set. From a practical viewpoint, gene ontology is a representation of something we know regarding a gene. GO addresses three domains: the cellular component, biological pathway, and molecular function. The terms in GO have a hierarchical structure and the GO graph has non-circular directed links. Each of the terms in GO has a relationship with certain genes. If we identify GO terms that have a relationship with the given DEG, we can assume that this DEG has characteristics that GO terms represent. To maximize the opportunities for identifying the hidden meanings of DEG characteristics (using a biological analysis process), an effective visualization and data management tool is indispensable.

In this paper, we propose the interactive visualization tool DegoViz (from DEG and GO), an analysis tool for a heatmap and gene ontology graph. DegoViz supports identifying a DEG from microarray data using custom threshold values from the user and visualizing the heatmap from the output of the DEG list and the corresponding GO graph. To allow biologists to discover new insight from this visualized data, DegoViz also provides an interaction technique between the visualized data of the heatmap and the GO graph. This includes an interactive response that when either genes on the heatmap or the GO terms on the GO graph are selected, the corresponding element reacts. In this manner, we can easily observe connections between groups of genes on the heatmap and the GO terms on the GO graph. We focus on the aesthetic approach, which is an important factor in data visualization for the recognition of complex data [4]. We expect that these new technical and graphic approaches to assist biologists determine new and meaningful insight.

DegoViz overcomes the previous problems by integrating the three vital functions into a single application (see Figure 1). It is optimized for DEG research and its corresponding GO terms. It is the first tool that proposes the display of a specific relationship between these items with real-time interaction. This facilitates the simultaneous management of the vital functions of biological analysis using interactive means. The proposed tools maximize the efficiency and convenience of the DEG analysis process.

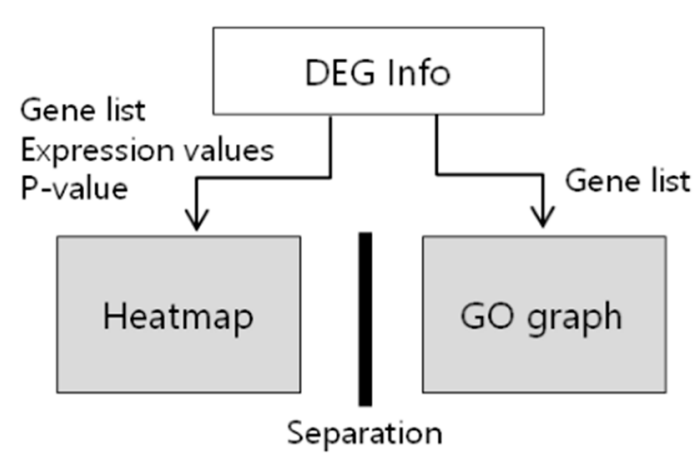

(a)

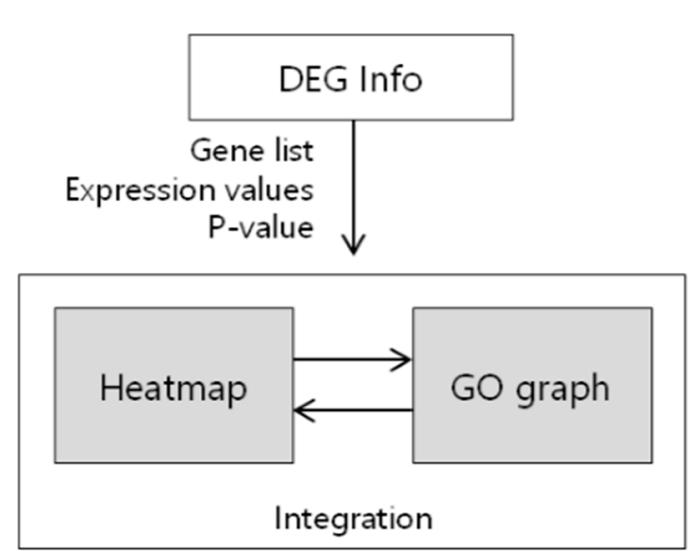

(b)

Figure 1. Comparison between previous DEG analysis and the proposed DegoViz tool. (a) Previous analysis process; (b) Proposed DegoViz tool.

\section{Materials and Methods}

\subsection{Related Works}

Currently, many tools have been proposed for analyzing DEGs and the corresponding GO terms. They can be categorized into three groups according to their purpose: (1) identifying a DEG from microarray data; (2) visualizing the corresponding GO from the DEG; and (3) exploring information related to a GO term. 
First, for identifying DEGs from the microarray data, some tools are available to obtain the desired result, including a single image heatmap visualizing the expression of the genes and organism [5-7]. Because they focus on statistical techniques, they do not provide a graphical user interface (GUI) for modifying the heatmap. Second, web-based applications for determining GO terms exist [8-10]. When there is user provided DEG input data, applications provide the corresponding GO terms with a visualized graph. However, the majority of these applications, such as GOrilla (see Figure 2) [10], are based on the graph drawing software graphViz [11], which only provides a single static image of the drawn graph. Force-directed graphs for visualization were proposed [12]. Several applications such as biNGO [13] are implemented based on Cytoscope [14], an effective tool for visualizing network relationships and providing user interaction. However, it does not provide the interactivity between integrated visualizations. Therefore, when users require additional information regarding a certain GO term, they must use another application for searching the GO library, such as QuickGO [15] or AmiGo [16]. These GO term searching applications provide specific information and relationships between GO terms.

In the process of biological research, from the data analysis of DEGs to viewing the related GO graph and specific terms, the three types of applications mentioned above must be utilized. Despite the importance of this ongoing process, due to its existence in distinct applications, there is a lack of efficiency and inconvenience in research and a low probability of finding new observations. Further, as previously mentioned, the technique in graph visualization depending on graph visualizing software $[11,14]$ is limited to drawing static images, which decreases the possibilities of application development for other purposes.

\subsection{Functions of DegoViz and Development Environment}

DegoViz is a window-based software and is an implemented version of a prior study regarding visualizing microarray and gene ontology analysis [17]. Each step of the implementation is completed to provide biologists with new insight and focuses on research efficiency and intuitive visualization. The program is structured in three modules: session management, data processing, and visualization (see Table 1). The session management module provides session-based data management. Working in a session saves the target microarray files, group information for the files, and species information. DegoViz accepts the CEL file format of the data measured by Affymetrix arrays. Users can also store threshold values such as $p$-values and fold-change values to limit the number of DEGs. If users perform the data processing step, the result is stored in the session folder and users are not required to perform the same data processing step in the future. The data processing module is programmed to determine the DEG list according to the defined threshold values. We use the affy and limma methods implemented in the affy and limma package in R $[18,19]$. After producing the DEG list, DegoViz searches for related GO terms using the clusterProfiler package in $\mathrm{R}$ [20]. Information regarding the DEG list and GO terms is stored for visualization. The visualization module draws an interactive heatmap and GO graph using the DEG list and related GO terms. Both the heatmap and GO graph are implemented in a real-time interactive manner, which allows users to observe the relationship between the DEGs and GO terms. The GO.db package in R [21] provides information regarding the hierarchy structure for the GO terms, which are illustrated in the GO graph. 


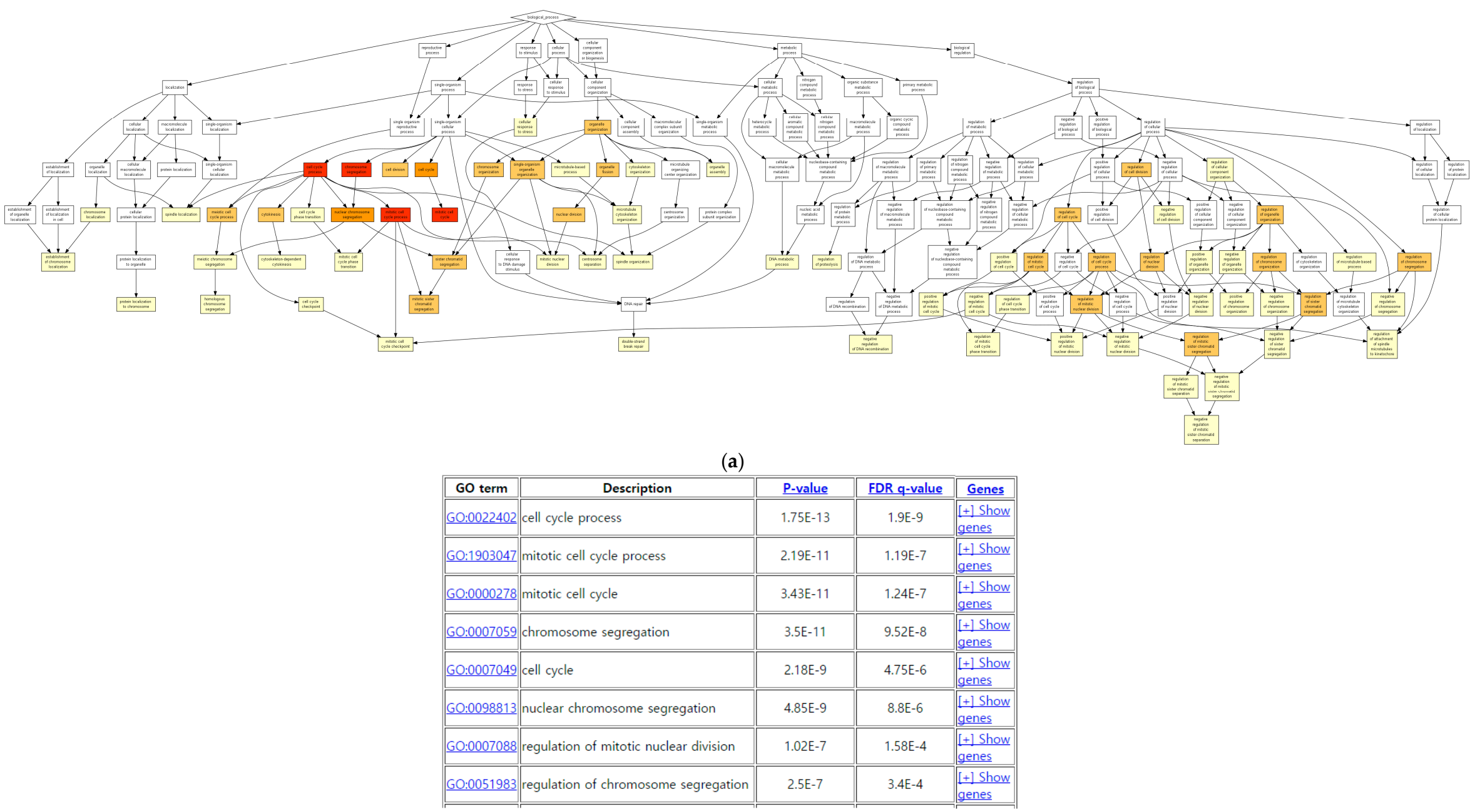

(b)

Figure 2. Example of GO result by Gorilla (http://cbl-gorilla.cs.technion.ac.il/). The upper part of the image is a visualization of the GO graph and the table below describes the enriched GO terms from the GO graph. 
Table 1. Summary of functions of the DegoViz tool.

\begin{tabular}{ll}
\hline \multicolumn{1}{c}{ Module } & \multicolumn{1}{c}{ Functions } \\
\hline \multirow{3}{*}{ Session management } & $\begin{array}{l}\text { Manage target microarray files } \\
\text { Store group information of microarray files } \\
\text { Store species information } \\
\text { Store results of data processing results } \\
\\
\text { Manage threshold values for determining DEGs }\end{array}$ \\
\hline \multirow{3}{*}{ Data processing } & Determine DEG list according to threshold values \\
& Identify GO terms related to DEGs \\
& Manage GO information including GO hierarchy structure \\
\hline \multirow{3}{*}{ Visualization } & Draw and manipulate heatmap \\
& Draw and manipulate GO graph \\
& Manage connection between heatmap and GO graph \\
\hline
\end{tabular}

The user interface of DegoViz is implemented in the Java language. The majority of the data processing is implemented in the R language, (http:/ / www.r-project.org), which uses subpackages in Bioconductor [22]. To communicate between $\mathrm{R}$ and Java, we use the RServe server engine [23]. To visualize the clustered data passed by $R$, we reconstruct the data into a directed acyclic graph structure; we use the jGprahT library [24]. Visualization implementation is completed with the Processing library [25], which is a strong tool for interactive visualization. Additional plugins are the toxiclibs (http://toxiclibs.org/) and ControlP5 (http://www.sojamo.de/libraries/controlP5/), which are used for the physical simulations and the GUI.

There are three important requisites that must be considered when designing the GO graph with the DEG list. First, the overall pathway of the ontology relationship must be easily recognizable. When biologists research the GO terms from the graph, they analyze them by following the relation path upward/downward between GO terms. It is important to have fluent work lines along the connection of the GO term nodes. Secondly, the enriched GO terms must be clearly perceptible to the researchers. Because the GO terms have special meaning and information in research, distinct from normal terms, they must be easily distinguishable from the other terms. Finally, a high perception of the relationship between the DEGs and the corresponding GO terms provides greater opportunities for identifying new interesting topics. Therefore, determining the number of significant results that are affected depends on the design of the DEG and the GO term relationship.

When visualizing the three design requisites together for effective biological research, we think of a modern subway route map design. First proposed by Harry Beck [26], the subway route map proposes an intuitive design for complex networks and has characteristics that are similar to our use of design requisites. First, the subway stations in the same line are linked continuously from start to end. The continuous line makes it easy to follow the path of multiple nodes. Further, the name of each station is labeled next to the station designation, which is outside the path. This manner of displaying provides a useful recognition of the linked nodes and separation of nodes and labels, which provides an additional element that can store information. We also introduce the concept of grid layout. Former studies have determined that from human study, users tend to layout graph data in an orthogonal shape [27]. We apply this information by placing and drawing the nodes in a grid position.

\section{Results}

With the rapid development of bioinformatics computing technology, visualizing large, complex biological data has become an important topic. Visualization facilitates determining new insight that was not developed using the former data. In this study, we propose two major visualizations: the DEG heatmap and the corresponding GO graph. The visualization provides focus in three areas. First, it enriches the intuition of complex data by converting text data to that which is visual. Then, it allows convenience in searching through the data using an interactive dashboard. Finally, it provides 
a relationship between the DEG and the GO term by allowing for the real-time interaction between the two visualizations.

\subsection{DEG Heatmap}

Microarray DEG data is a set of differentially expressed genes. The data set increases to an enormous scale when the number of samples being compared is large or the DEG is identified in large numbers. For this reason, the proposed common method of presenting gene expression in biomedical science is the use of a heatmap. Each sample of the gene is color-coded for different strengths, which makes it easier to compare the expression levels from the overall expression map.

We follow the most common approach of the previous heatmap visualization method and provide additional functionality (see Figure 3). The proposed implemented heatmap is interactive, not static; users can zoom in/out on the heatmap; this assists in the viewing of the affected genes inside the hundreds of genes in the heatmap. Moreover, users can display various gene IDs such as a prove ID, gene symbol, and Entriz gene ID on the heatmap. The proposed heatmap provides gene searching using any ID type. Finally, by positioning the mouse in a specific location of the DEG list, users can view information that is more detailed, such as the gene expression value, which is a description concerning pointed genes. The web-based SVM (Smart Virtual Machine) interpreter executes the contents through a stack-based calculation. To accomplish this, the interpreter loads the SEF file and executes the relevant command. The results are reflected in the memory model and interpreter status.

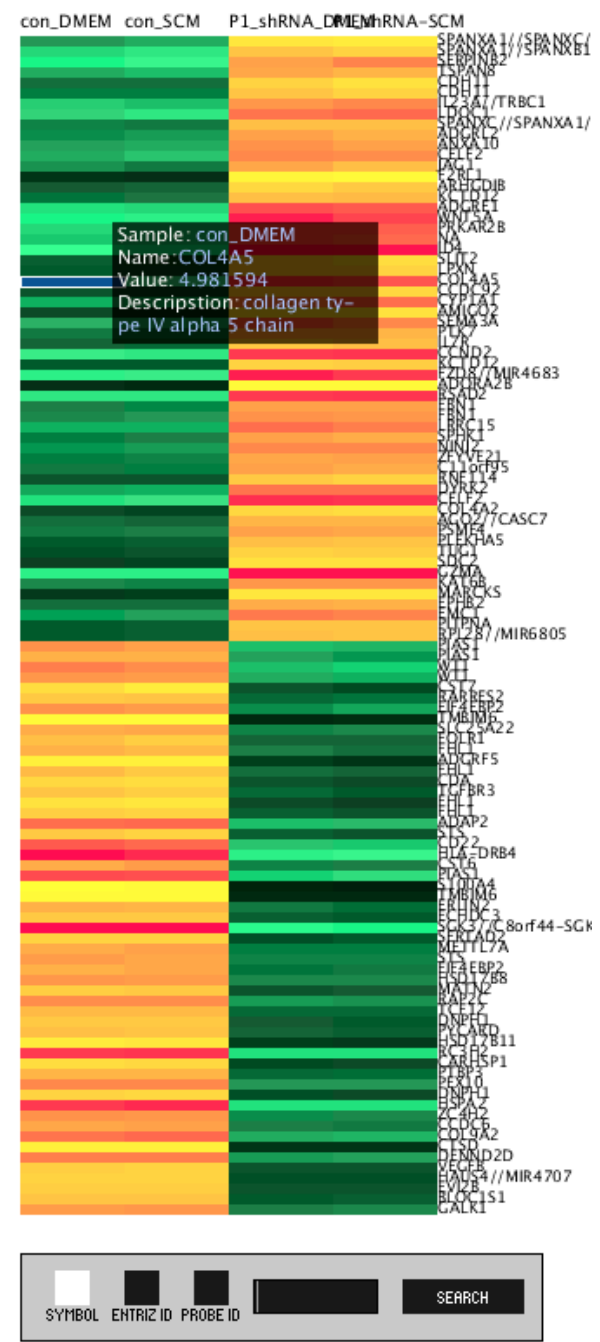

Figure 3. Visualization of the DEG Heatmap. 


\subsection{Gene Ontology Graph}

Gene ontology has a hierarchical data structure, where each term can have multiple parents and multiple children. A directed acyclic graph (DAG) is commonly used for the GO graph. GO terms and their relationships are expressed by nodes and edges. The primary difficulty in GO graph visualization is displaying a large number of edges. Because GO terms have eight types of different relationships between them, a GO graph displays considerably more edges than a normal graph [28]. In our implementation, we visualize a GO graph that is based on the DAG drawing strategy proposed by AT\&T [29]. The strategy proposes minimizing edge crossing by utilizing a heuristic approach.

The GO graph visualization in DegoViz has diverse properties. Each GO term displays the connections with neighbors with its annotation (see Figure 4). Enriched GO terms are color-coded with their $p$-value and display the corresponding DEG collection, which is designated within a circle. If GO terms share the same DEG, then DegoViz provides interactive facilities similar to the heatmap. Users can review detailed information on the specific GO term, including the corresponding DEG list, by simply placing the mouse over it. When the GO term is clicked, all of the parent GO terms between the root GO terms are highlighted and vice versa. This allows the users to easily follow the path of all related terms from the overall graph. We also propose the display controller (on the right side of the window). This allows the display of only those elements set by the user. This facilitates the clear recognition of a complex relationship between GO terms because users can select a certain type of relationship for display. When corresponding GO terms appear on large-scale graph data, it is difficult to display and understand the details from the "big" picture. For a more user-friendly data search, we provide a zoom in/out and screen move function, allowing users to easily move to a position on the overall graph and display the specific relationship of the GO terms.
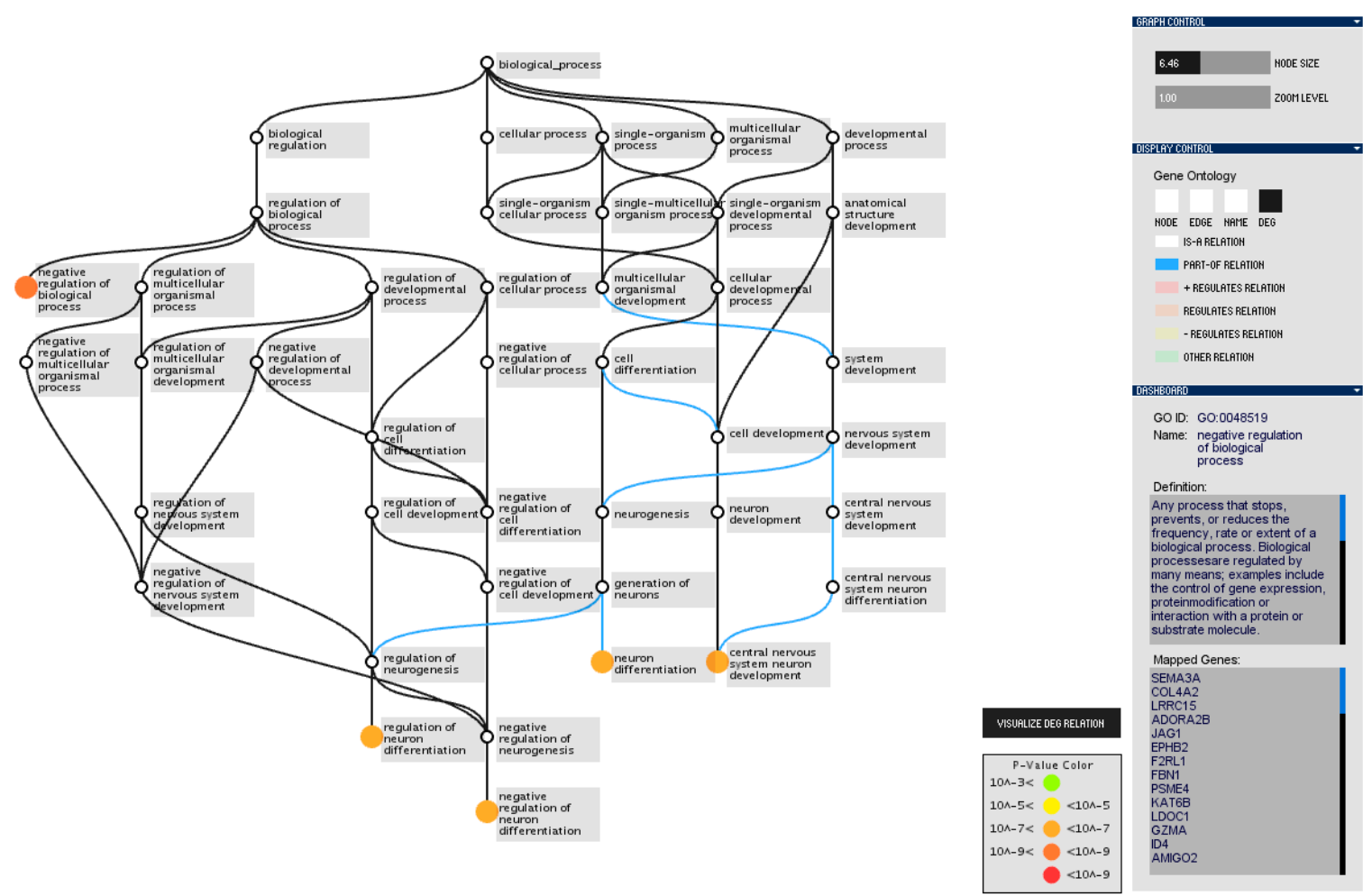

Figure 4. Visualization of the gene ontology graph.

To consider the relationship between different enriched GO terms that share the same DEG, we introduce two additional visualization tools for the relation between a DEG and corresponding GO terms. The first graph displays how GO terms share their corresponding DEG sets (see Figure 5). 
Over the enriched gene ontology, the number of corresponding DEGs is assigned in green. A green line between two GO terms means that they share the same genes. The assigned number on the line indicates the number of shared genes. For example, in Figure 5, the graph indicates that two GO terms, "neuron differentiation" and "central nervous system neuron development", share five DEGs, where each has 13 and five corresponding DEGs. This means that all of the DEGs from the latter are included in the former ontology.

Another graph indicates how an individual DEG relates to the corresponding GO terms (see Figure 6). It displays specific DEGs that are linked to multiple GO terms. We implement this feature using multidimensional scaling (MDS) $[30,31]$ in circular space. The MDS method places the DEGs, the green dots in the graph in Figure 6, at the optimal position nearest to the enriched GO terms to which they belong. This allows users to recognize the similarity of the DEG set, which relates to similar GO terms. For example, by reviewing Figure 6, we can observe that the four DEGs located in the center are linked to all of the enriched GO terms, which means that these genes belong to all of the enriched GO terms in the graph. Further, we can observe that all DEGs that belong to ontology "negative regulation of neuron differentiation" and "central nervous system neuron development" are shared with the three other enriched GO terms. For both graphs, user interaction is available to search for information that is more specific.
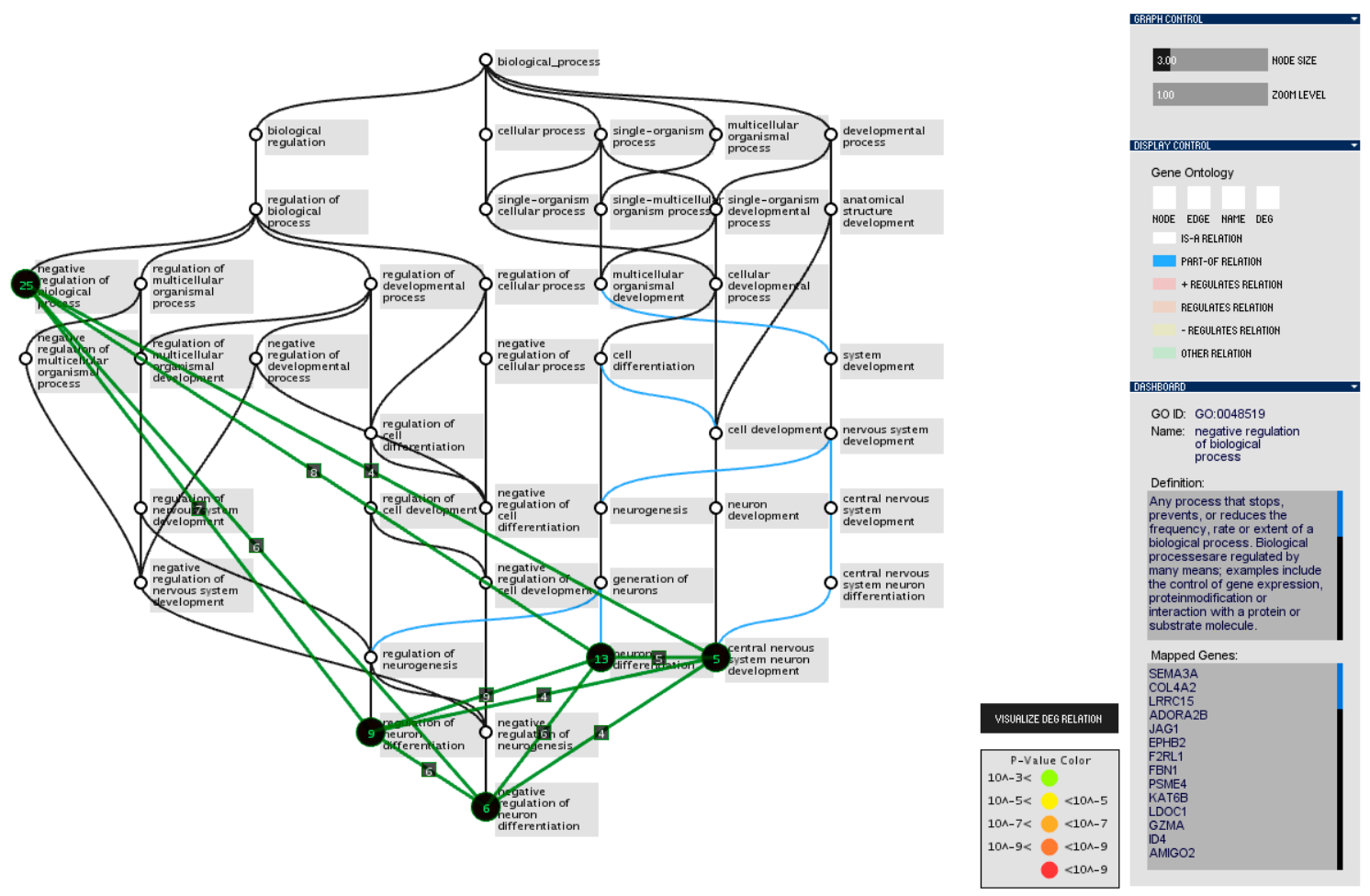

Figure 5. Visualization of the number of DEGs shared between enriched GO terms. 


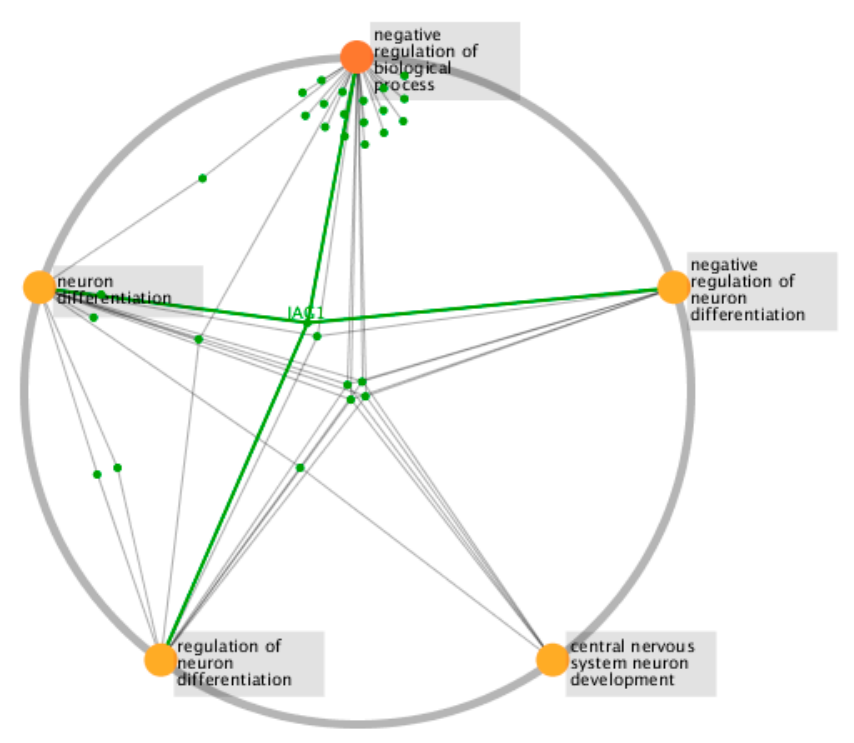

Figure 6. Visualization of the DEG and GO relationship.

\subsection{Interaction Design between Heatmap and Gene Ontology Graph}

As mentioned previously, integrating the DEG heatmap and gene ontology graph provides the relationship between the DEG and the corresponding GO terms to enhance the probability of identifying new insight into biological meaning. For this purpose, DegoViz implements a real-time connection between the DEG heatmap and the GO graph. From the heatmap view, when a set of DEGs on the heatmap is selected, the corresponding GO terms on the GO graph respond. Similarly, from the GO graph view, when users select a specific enriched GO term from the GO graph, its corresponding genes on the heatmap respond. Figure 7 illustrates how interaction works between the heatmap and GO graph. In Figure 7a, eight DEGs are selected from the heatmap and all the enriched GO terms that are related to those selected DEGs are identified in the GO graph in red. Moreover, the selected DEGs are highlighted in an additional visualization, as illustrated in Figure 7c. In Figure 7b, the ontology "neuron differentiation" is selected from the GO graph and the DEGs that are related to this ontology are highlighted in the heatmap by a blinking blue color.

This functionality is new to the proposed tool. In the previous analysis process, determining the DEGs and the posterior analysis for the DEGs was separate (see Figure 1). The heatmap for the DEG provides a wealth of information: the difference in the expression level between genes, $p$-values and fold change (FC) values, and up/down regulated genes between sample groups. However, only the list of gene names is used for posterior analysis. The posterior analysis tool omits the information that the heatmap displays (see Figure 1a). It restricts users to the biological insight that the DEG list contains. Therefore, the proposed DegoViz attempts to retain the valuable information in the heatmap during the posterior analysis (see Figure 1b). Users can review the related GO terms with the overall DEG list. They can also examine the related GO terms for only upregulated genes or affected genes in the DEG list. 

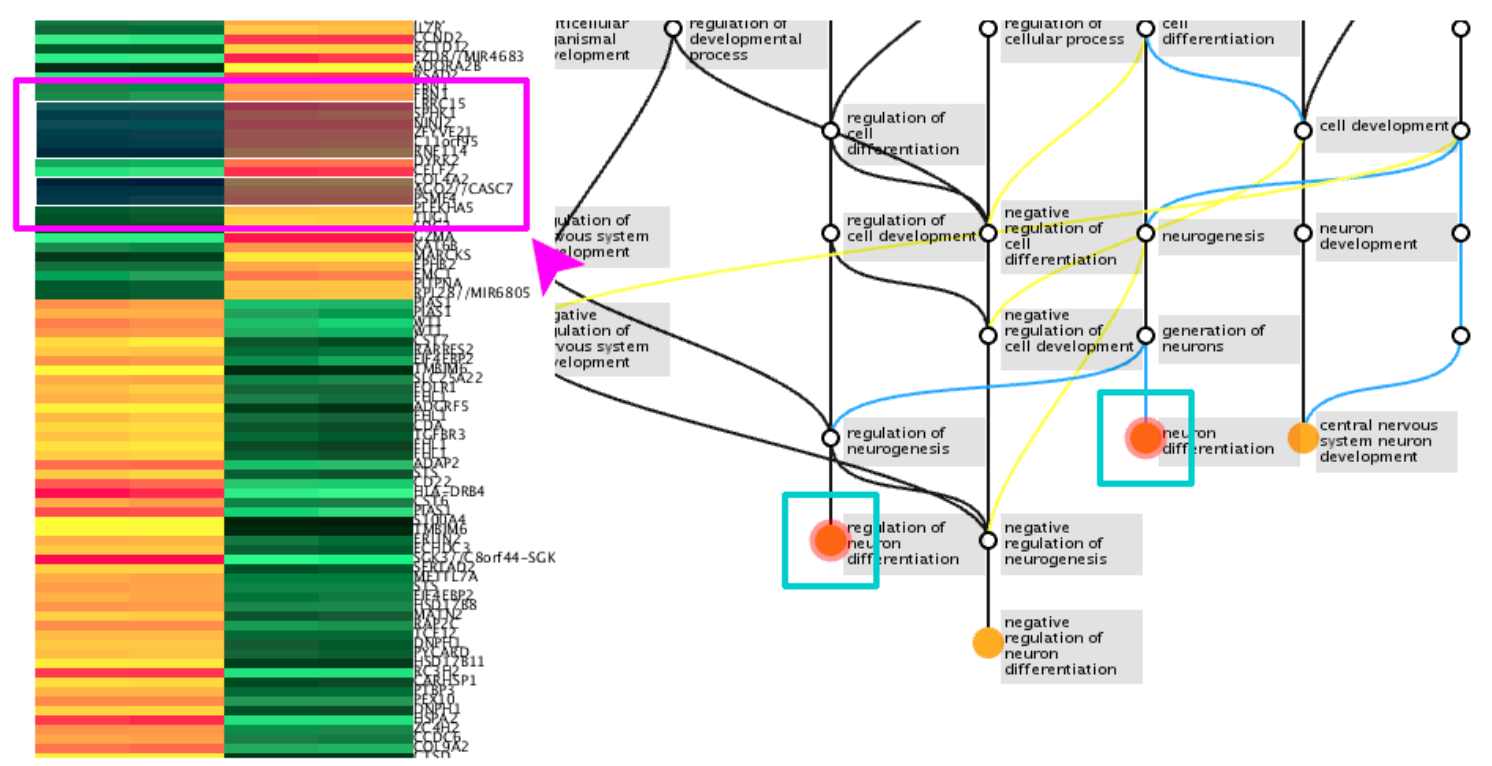

(a)

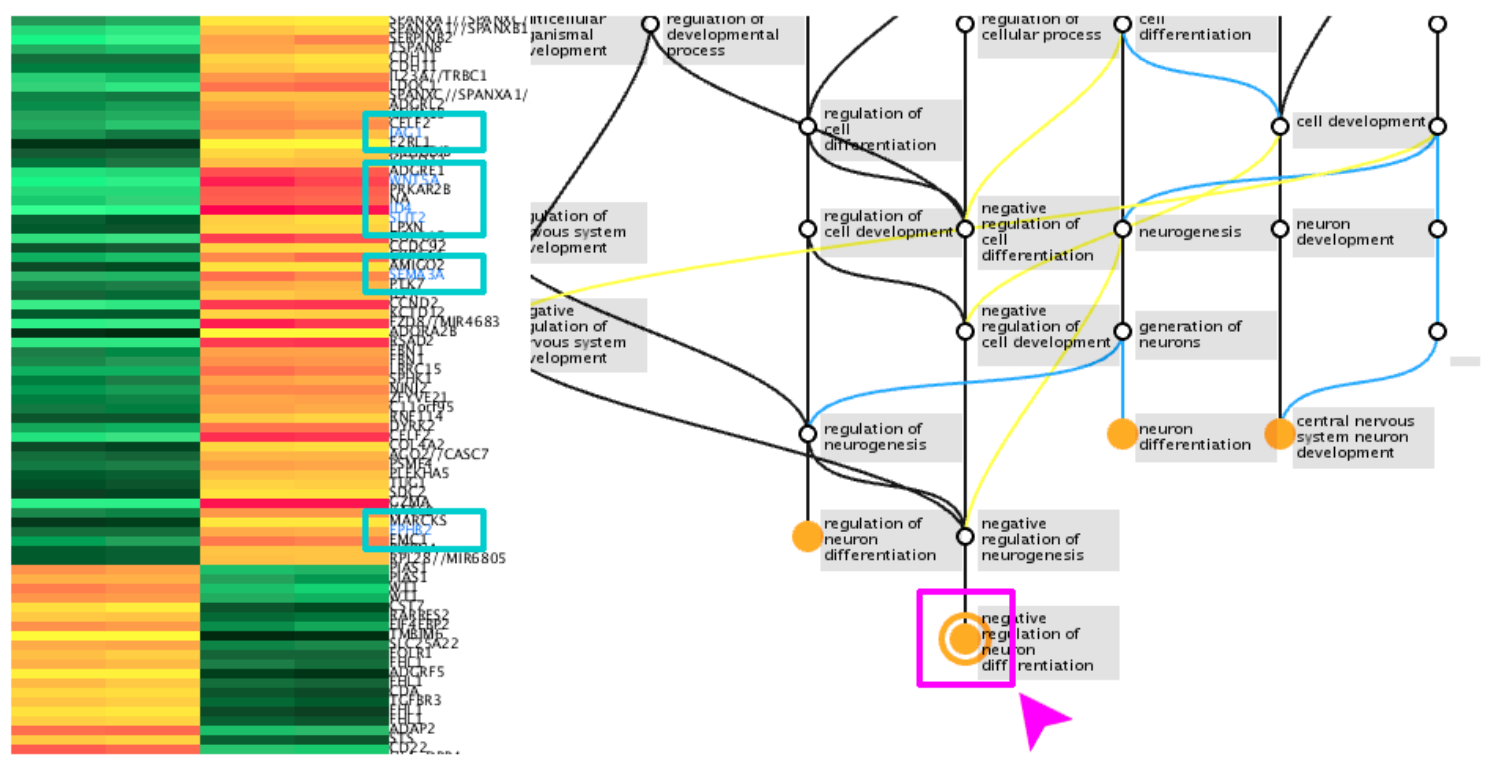

(b)

Figure 7. Cont. 


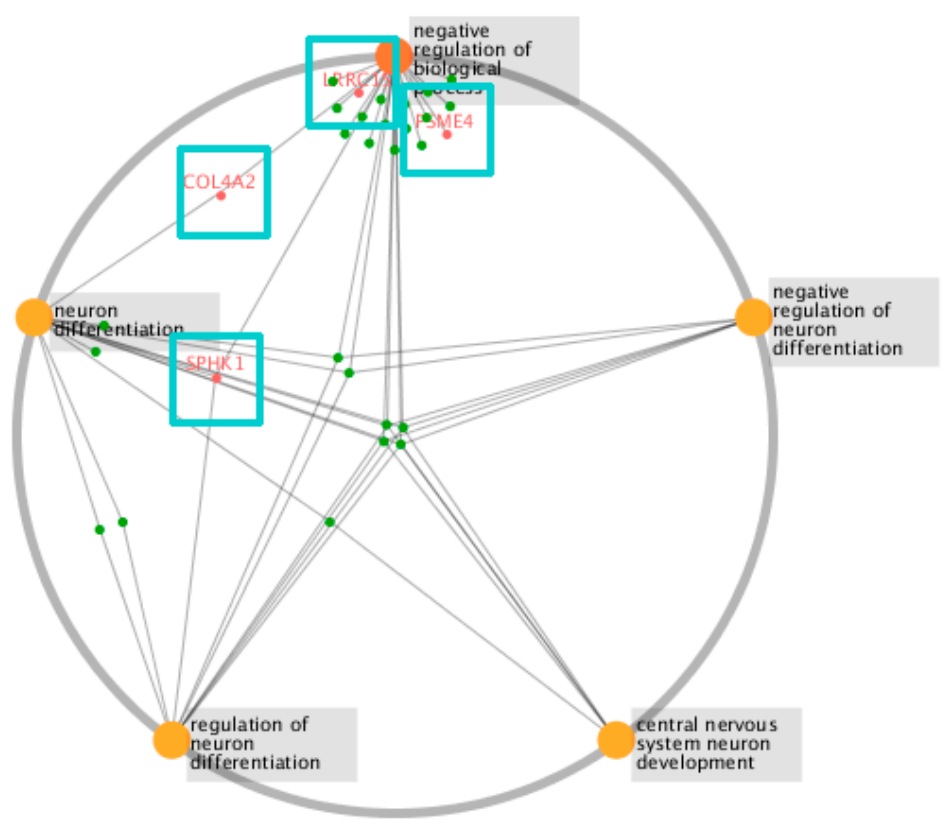

(c)

Figure 7. Interaction between the heatmap and gene ontology graph. (a) Response (highlighted cyan) from the GO graph when the DEG set is selected (highlighted magenta); (b) Response (highlighted cyan) from the heatmap of GO term selection (highlighted magenta); (c) Response (highlighted cyan) from the gene and gene ontology relation when DEG is selected from the heatmap.

\section{Discussion}

DegoViz focuses on the aesthetic aspect of visualizing the GO graph. In previous DEG analysis work, the GO graphs were typically visualized in a hierarchical tree, which was not significantly different from the work proposed by GOrilla (see Figure 2). In this visual representation, each of the GO terms is presented as a primitive shaped node. Then, they are linked with directional arrow lines and color-coded based on the expression values. The image displays all of the essential information that must be expressed; however, the application of its flat color and stiff shape seriously limits the potential of providing a high perception. Other works proposed the force-directed graph for visualization. This radial shaped method of visualizing has more weaknesses than strengths in GO graph visualization. Although it has the efficiency of saving space when drawing a tree structure, the complexity of the GO term relations is difficult to recognize when drawn in a forced-directed graph. Furthermore, it is difficult to see the hierarchical order and the overall flow of the ontology path.

In the biological process, genes interact when joined together. This means that life phenomena are not dependent on a single gene; rather, they are dependent on a group of genes. An important task is to determine the biological meaning of a particular group of genes. In this paper, we proposed an integrated visualization tool to facilitate the determination of this meaning and develop biological insight from a DEG list. The proposed prototype supports the biological process of GO. Our next version may contain a cellular component and molecular function. Further, we may include an advanced function such as a DEG selection tool.

Author Contributions: Kyungwon Lee and Sejong Oh conceived and designed the experiments; Somyung Oh performed the experiments; Junghyeon Ha analyzed the data; Junghyeon Ha contributed reagents/materials/analysis tools; Somyung Oh and Sejong Oh wrote the paper.

Conflicts of Interest: The authors declare no conflict of interest. 


\section{References}

1. Altman, N. Replication, variation and normalization in microarray experiments. Appl. Bioinform. 2005, 4, 33-44. [CrossRef]

2. Wang, Z.; Gerstein, M.; Snyder, M. RNA-Seq: A revolutionary tool for transcriptomics. Nat. Rev. Genet. 2009, 10, 57-63. [CrossRef] [PubMed]

3. Gene Ontology Consortium. The Gene Ontology (GO) database and informatics resource. Nucleic Acids Res. 2004, 32 (Suppl. 1), D258-D261.

4. Cawthon, N.; Moere, A.V. The effect of aesthetic on the usability of data visualization. In Proceedings of the 11th International Conference on Information Visualization, IV'07, Zurich, Switzerland, 4-6 July 2007.

5. Shannon, W.; Robert, C.; Jill, D. Analyzing microarray data using cluster analysis. Pharmacogenomics 2003, 4, 41-52. [CrossRef] [PubMed]

6. Johnson, W.E.; Cheng, L.; Ariel, R. Adjusting batch effects in microarray expression data using empirical Bayes methods. Biostatistics 2007, 8, 118-127. [CrossRef] [PubMed]

7. Xia, Q.; Cheng, D.; Duan, J.; Wang, G.; Cheng, T.; Zha, X.; Liu, C.; Zhao, P.; Dai, F.; Zhang, Z.; et al. Microarray-based gene expression profiles in multiple tissues of the domesticated silkworm, Bombyx mori. Genome Biol. 2007, 8, R162. [CrossRef] [PubMed]

8. Al-Shahrour, F.; Ramón, D.; Joaquín, D. FatiGO: A web tool for finding significant associations of Gene Ontology terms with groups of genes. Bioinformatics 2004, 20, 578-580. [CrossRef] [PubMed]

9. Doniger, S.W.; Salomonis, N.; Dahlquist, K.D.; Vranizan, K.; Lawlor, S.C.; Conklin, B.R. MAPPFinder: Using Gene Ontology and GenMAPP to create a global gene-expression profile from microarray data. Genome Biol. 2003, 4, R7. [CrossRef] [PubMed]

10. Eden, E.; Navon, R.; Steinfeld, I.; Lipson, D.; Yakhini, Z. GOrilla: A tool for discovery and visualization of enriched GO terms in ranked gene lists. BMC Bioinform. 2009, 10, 48. [CrossRef] [PubMed]

11. Ellson, J.; Gansner, E.; Koutsofios, L.; North, S.C.; Woodhull, G. Graphviz-Open source graph drawing tools. In Proceedings of the International Symposium on Graph Drawing, Vienna, Austria, 23-26 September 2001.

12. Salazar-Henao, J.E.; Schmidt, W. An inventory of nutrient-responsive genes in arabidopsis root hairs. Front. Plant Sci. 2016, 7, 1-12. [CrossRef] [PubMed]

13. Maere, S.; Heymans, K.; Kuiper, M. BiNGO: A Cytoscape plugin to assess overrepresentation of gene ontology categories in biological networks. Bioinformatics 2005, 21, 3448-3449. [CrossRef] [PubMed]

14. Shannon, P.; Markiel, A.; Ozier, O.; Baliga, N.S.; Wang, J.T.; Ramage, D.; Amin, N.; Schwikowski, B.; Ideker, T. Cytoscape: A software environment for integrated models of biomolecular interaction networks. Genome Res. 2003, 13, 2498-2504. [CrossRef] [PubMed]

15. Binns, D.; Dimmer, E.; Huntley, R.; Barrell, D.; O'donovan, C.; Apweiler, R. QuickGO: A web-based tool for Gene Ontology searching. Bioinformatics 2009, 25, 3045-3046. [CrossRef] [PubMed]

16. Carbon, S.; Ireland, A.; Mungall, C.J.; Shu, S.; Marshall, B.; Lewis, S. Web Presence Working Group. AmiGO: Online access to ontology and annotation data. Bioinformatics 2009, 25, 288-289. [CrossRef] [PubMed]

17. Oh, S.; Ha, J.; Lee, K.; Oh, S. Development of Integrated Visualization Tool for Differentially Expressed Genes and Gene Ontology Analysis. Int. J. Image Signal Syst. Eng. 2017, 1, 1-6. [CrossRef]

18. Gautier, L.; Cope, L.; Bolstad, B.M.; Irizarry, R.A. Affy-Analysis of Affymetrix GeneChip data at the probe level. Bioinformatics 2004, 20, 307-315. [CrossRef] [PubMed]

19. Ritchie, M.E.; Phipson, B.; Wu, D.; Hu, Y.; Law, C.W.; Shi, W.; Smyth, G.K. limma powers differential expression analyses for RNA-sequencing and microarray studies. Nucleic Acids Res. 2015, 43, e47. [CrossRef] [PubMed]

20. Yu, G.; Wang, L.G.; Han, Y.; He, Q.Y. clusterProfiler: An R package for comparing biological themes among gene clusters. Omics 2012, 16, 284-287. [CrossRef] [PubMed]

21. Carlson, M. A Set of Annotation Maps Describing the Entire Gene Ontology. Available online: https: / / bioconductor.org/packages/release/data/annotation/html/GO.db.html (accessed on 25 May 2017).

22. Gentleman, R.C.; Carey, V.J.; Bates, D.M.; Bolstad, B.; Dettling, M.; Dudoit, S.; Ellis, B.; Gautier, L.; Ge, Y.; Gentry, J.; et al. Bioconductor: Open software development for computational biology and bioinformatics. Genome Biol. 2004, 5, R80. [CrossRef] [PubMed]

23. Urbanek, S. Rserve-A Fast Way to Provide R Functionality to Applications. In Proceedings of the 3rd International Workshop on Distributed Statistical Computing, Vienna, Austria, 20-22 March 2003. 
24. Naveh, B.; Sichi, J.V. JGraphT a Free Java Graph Library. Available online: http://jgrapht.org/ (accessed on 11 December 2016).

25. Reas, C.; Fry, B. Processing: Programming for the media arts. AI SOCIETY 2006, 20, 526-538. [CrossRef]

26. Garland, K. Mr Beck's Underground Map; Capital Transport Publishing: London, UK, 1994.

27. Kieffer, S.; Dwyer, T.; Marriott, K.; Wybrow, M. Hola: Human-like orthogonal network layout. IEEE Trans. Vis. Comput. Gr. 2016, 22, 349-358. [CrossRef] [PubMed]

28. Dessimoz, C.; Škunca, N. The Gene Ontology Handbook; Humana Press: New York, NY, USA, 2016.

29. Gansner, E.R.; Koutsofios, E.; North, S.C.; Vo, K.P. A technique for drawing directed graphs. IEEE Trans. Softw. Eng. 1993, 19, 214-230. [CrossRef]

30. Kruskal, J.B. Multidimensional scaling by optimizing goodness of fit to a nonmetric hypothesis. Psychometrika 1964, 29, 1-27. [CrossRef]

31. Kruskal, J.B.; Wish, M. Multidimensional Scaling. In Sage University Paper Series on Quantitative Applications in the Social Sciences; Sage Publications: Beverly Hills, CA, USA, 1978.

(C) 2017 by the authors. Licensee MDPI, Basel, Switzerland. This article is an open access article distributed under the terms and conditions of the Creative Commons Attribution (CC BY) license (http:/ / creativecommons.org/licenses/by/4.0/). 